\title{
Analyse de la construction de la vulnérabilité des ménages du système irrigué de Guédé au nord du Sénégal
}

\author{
Anne Sila ${ }^{1,2}$, Françoise Gérard ${ }^{1,2}$, William's Daré ${ }^{1,2}$, Alpha Ba $^{3, *}$, Elhadj Faye ${ }^{4}$, \\ Amandine Adamczewski ${ }^{5,6}$ et François Bousquet ${ }^{1,2}$ (1) \\ ${ }^{1}$ CIRAD, UMR SENS, 34398 Montpellier, France \\ 2 SENS, Univ Montpellier CIRAD, IRD, Université Paul Valéry Montpellier 3, Montpellier, France \\ ${ }^{3}$ ESR/ENSA, Université de Thiès, Thiès, Sénégal \\ ${ }^{4}$ ENDA PRONAT, Dakar, Sénégal \\ ${ }^{5}$ CIRAD, UMR G-EAU, Montpellier, France \\ ${ }^{6}$ G-EAU, Univ Montpellier, AgroParisTech, CIRAD, INRAE, Institut Agro, IRD, Montpellier, France
}

\begin{abstract}
Résumé - Malgré l'amélioration qu'ils apportent à la production agricole, les périmètres irrigués génèrent des risques sociaux, économiques et écologiques importants, qui questionnent leurs impacts effectifs sur la vulnérabilité sociale, économique, environnementale et alimentaire des populations bénéficiaires. La réflexion sur la construction de la vulnérabilité mobilisée dans le cadre de cet article cherche à analyser, dans une perspective socio-historico-économique, comment les processus, les changements et les perturbations dans le temps et dans l'espace peuvent expliquer les mécanismes à la source des vulnérabilités actuelles des ménages installés autour du système irrigué de la cuvette de Guédé au Sénégal. Les résultats mettent en évidence une vulnérabilité différentielle des types de ménages à plusieurs facteurs de stress. Cette vulnérabilité est fonction de la faiblesse des surfaces cultivées et des rendements, des coûts de production élevés ou des difficultés d'accès à des activités extra-agricoles, mais aussi de la période et du lieu d'installation des ménages, qui définissent partiellement leur dotation en ressources, et ainsi leur capacité à faire face à des conditions changeantes. Pour fournir une réflexion pluridisciplinaire, systémique et nuancée sur la vulnérabilité des ménages agricoles du système irrigué de Guédé, il est important de comprendre les mécanismes qui fondent la construction et l'évolution de la vulnérabilité et de voir comment leurs interactions dynamiques peuvent réduire ou renforcer les vulnérabilités existantes ou créer de nouvelles vulnérabilités.
\end{abstract}

Mots-clés : vulnérabilité / adaptation / système irrigué / typologie / Guédé

\begin{abstract}
Analysis of the construction of the vulnerability of households in the irrigated system of Guédé in northern Senegal. Despite the improvement in agricultural production, irrigated schemes generate significant social, economic and ecological risks that raise questions about their actual impacts on the social, economic, environmental and food vulnerability of beneficiary populations. The reflection on the construction of vulnerability mobilized in this paper seeks to analyze, from a socio-historical and economic perspective, how the processes, changes and disruptions in time and space can explain the mechanisms at the source of the current vulnerabilities of households settled around the irrigated system of the Guédé basin in Senegal. The results highlight a differential vulnerability of households to several stressors. This vulnerability is a function of the small amount of land cultivated and yields, high production costs or difficulties in accessing off-farm activities, but also of the period and location of settlement of households, which partially define their resource endowment, and thus their capacity to cope with changing conditions. To provide a multidisciplinary, systemic and nuanced reflection on the vulnerability of agricultural households in the Guédé irrigated system, it is important to understand the mechanisms that underlie the construction and evolution of vulnerability and to see how their dynamic interactions can reduce or reinforce existing vulnerabilities or create new vulnerabilities.
\end{abstract}

Keywords: vulnerability / adaptation / irrigated system / typology / Guédé

\footnotetext{
*Auteur correspondant : annejeanne91@gmail.com
} 


\section{Introduction}

$\mathrm{Au}$ cours des dernières décennies, l'irrigation s'est développée en Afrique sahélienne en réponse aux contraintes hydro-climatiques de la région. Son essor s'est intensifié suite aux épisodes de sécheresse entre 1970 et 1980 . Depuis la crise alimentaire de 2008, elle connaît un regain d'intérêt de la part des bailleurs de fonds internationaux et des États comme un moyen d'assurer la sécurité alimentaire et d'augmenter le niveau de vie des populations. Dans la vallée du fleuve Sénégal, plusieurs travaux (Le Roy, 2006; Schmitz, 2008) montrent que malgré l'amélioration apportée à la production agricole par les infrastructures irriguées, celles-ci ne répondent pas toujours aux espoirs suscités et sont susceptibles d'accroître la vulnérabilité économique, sociale, politique ou environnementale des agriculteurs.

Ce travail s'inscrit dans la problématique générale de la vulnérabilité des ménages agricoles qui cultivent dans des périmètres irrigués. Selon Turner et al. (2003), la vulnérabilité est la mesure dans laquelle un système, un sous-système ou un composant du système est susceptible de subir des dommages en raison de son exposition, de sa sensibilité et de la manière dont il est susceptible de réagir et de mettre en œuvre des stratégies qui favorisent ses capacités à s'adapter et à faire face à des chocs.

Nous considérons la vulnérabilité comme un processus dynamique qui relève de l'étude des interrelations entre, d'une part, l'exposition à des aléas environnementaux et la sensibilité des socio-écosystèmes à ces aléas, et d'autre part, les spécificités des configurations et des dynamiques socioéconomiques locales dans leurs capacités d'adaptation (Turner et al., 2003 ; Spiegelberger et al., 2018).

Beaucoup de travaux sur la vulnérabilité des ménages sont concentrés sur l'impact de variables climatiques ou sur le lien avec la pauvreté. Ce focus masque cependant la vulnérabilité à d'autres facteurs de stress importants comme des stress agro-environnementaux, des politiques changeantes, la libéralisation d'un marché ou la perte d'interactions sociales (O’Brien et al., 2009). En Afrique subsaharienne, des travaux récents montrent que la vulnérabilité des ménages est également causée par une diversité de facteurs biophysiques, socio-économiques, culturels et politiques (Rurinda et al., 2014). Bien que les études sur la vulnérabilité se soient multipliées (Janssen et al., 2006), très peu d'entre elles ont cherché à combiner les facteurs socio-économiques avec une perspective historique pour mieux comprendre les contextes et les racines de la vulnérabilité (Bankoff, 2004).

L'objectif de cet article est d'analyser la vulnérabilité différentielle des ménages face aux transformations socioenvironnementales du système irrigué de Guédé au nord du Sénégal, où fut construit l'un des premiers périmètres irrigués $\mathrm{du}$ pays. À travers une approche pluridisciplinaire et systémique couplant la socio-histoire et l'économie, nous questionnons les facteurs historiques et socio-économiques, et nous examinons leurs liens dans la construction, l'évolution et la différenciation de la vulnérabilité des ménages du système irrigué de Guédé.

\section{Matériel et méthodes}

\subsection{Terrain de recherche}

L'étude porte sur le système irrigué de Guédé, situé dans le département de Podor dans la moyenne vallée du fleuve Sénégal (Fig. 1). Le climat sahélien se caractérise par une faiblesse et une irrégularité des précipitations avec une moyenne annuelle de $300 \mathrm{~mm}$ (Bruckmann, 2018). Au sein de la zone d'étude, les agriculteurs combinent leurs activités de production autour de l'agriculture irriguée, pluviale et de décrue, ainsi que de l'élevage.

Le système irrigué étudié est principalement constitué du grand périmètre irrigué de la cuvette de Guédé Chantier (595 ha). Mis en place à la période coloniale, il a connu une trajectoire d'aménagements et de restructurations en plusieurs périodes. Cette cuvette polarise autour d'elle cinq villages (Guédé Chantier, Guédé Village, Agnam Tonguel, Madina Fresbé et Lérabé). Ce système irrigué comprend aussi le périmètre irrigué villageois (PIV) de Lérabé (30 ha), le PIV de Guédé Village (200 ha) et une diversité de périmètres irrigués privés aménagés par des particuliers, pouvant aller de 1 à 30 ha.

\subsection{Collecte et analyse des données}

\subsubsection{Collecte des données socio-historiques par entretien et consultation des archives}

Entre avril et mai 2019, nous avons conduit 22 entretiens semi-structurés, orientés sur l'histoire de l'agriculture et des aménagements dans la zone, auprès de producteurs dans les différents périmètres irrigués et non irrigués, de personnalités de l'encadrement et d'organisations non gouvernementales qui interviennent dans la zone. Ces entretiens étaient combinés à des récits de vie où les personnes interrogées racontaient les évènements marquants vécus ou rapportés.

Puis, en juin 2019, en utilisant la méthode boule de neige (Combessie, 2007), nous avons interrogé 25 ménages répartis dans les 5 villages pour connaître la trajectoire de leurs exploitations. Le choix des ménages s'est fait en privilégiant le choix d'un chef de ménage ayant au minimum entre 45 et 50 ans, critère que nous avons défini pour nous assurer de recueillir les perceptions et les pratiques sur le long terme, tout en veillant à ce qu'elles soient vécues par la personne qui les raconte.

Pour avoir un maximum de profondeur historique, ces données ont été combinées avec une analyse de ressources bibliographiques issues de la littérature grise et des archives nationales. À partir des évènements marquants vécus ou relatés par les personnes interrogées et des données de la littérature grise, nous avons reconstitué les grandes périodes de l'évolution historique, tant dans les ruptures et les crises, que dans les solutions apportées.

\subsubsection{Collecte des données socio-économiques par questionnaire}

Pour compléter ces premières données, des enquêtes socioéconomiques ont été réalisées entre janvier et mars 2020 sur un échantillon de 206 ménages répartis sur les 5 villages, soit 


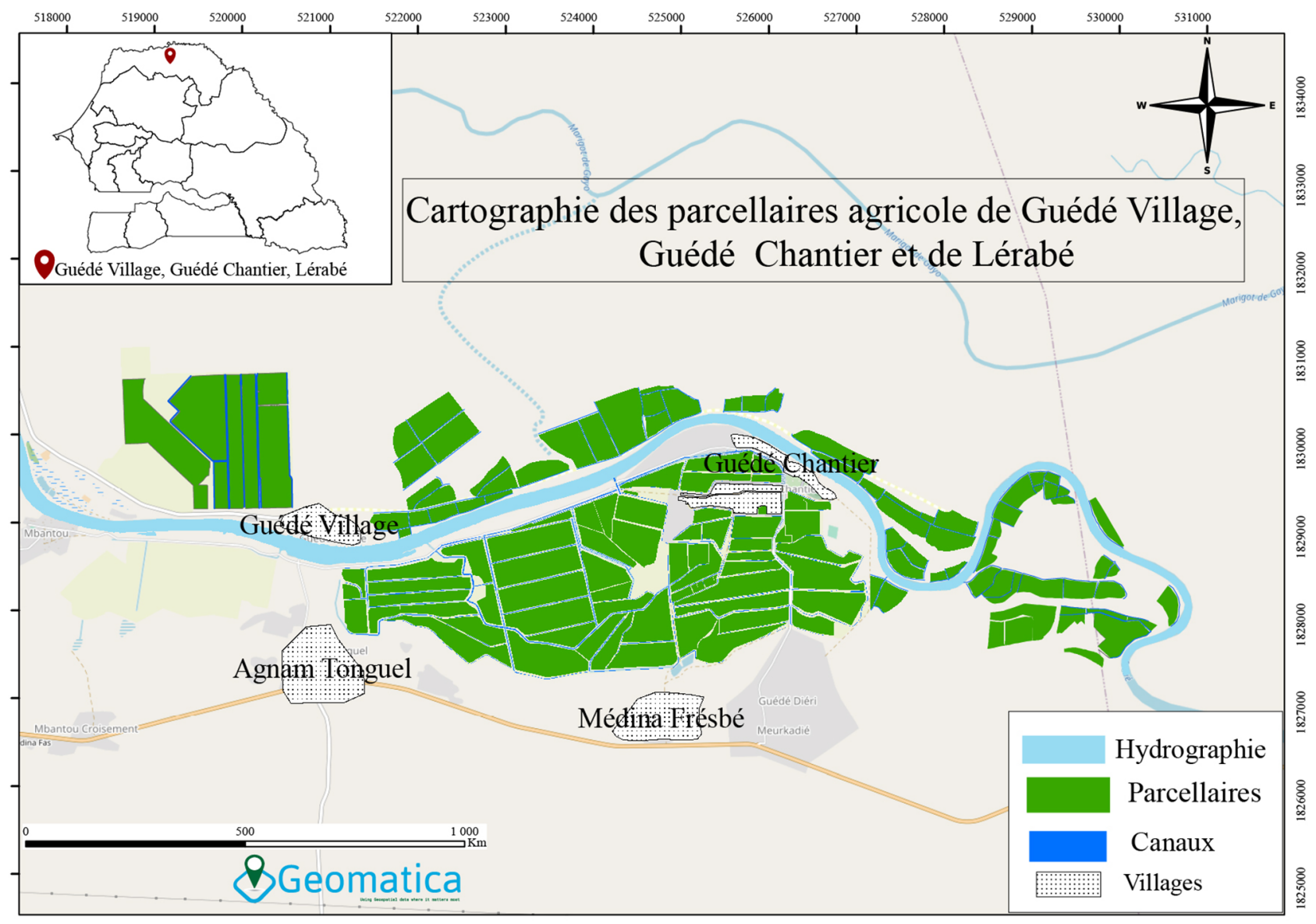

Fig. 1. Localisation de la zone d'étude.

Fig. 1. Study area location.

$17 \%$ de la population totale. Le questionnaire comprenait des questions quantitatives et qualitatives semi-ouvertes et fermées.

Le nombre de ménages à interroger par village a été défini par un système de quotas, et un pas de sondage de 3 a été appliqué. Ainsi, à partir d'un point de repère stratégique dans le village, le premier ménage à interroger a été identifié, et en partant de ce ménage repère, les autres ménages à interroger furent choisis toutes les trois maisons, en suivant le sens des aiguilles d'une montre.

L'approche qui a prévalu à l'élaboration du questionnaire socio-économique est celle des moyens d'existence en zone rurale (Chambers et Conway, 1991; Ellis, 2000), qui utilise le ménage comme unité d'analyse. Différentes variables ont été collectées pour décrire les dotations en facteurs de production, les caractéristiques historiques et géographiques du ménage, ainsi que ses différentes sources de revenus. D'autres variables comme les facteurs de stress identifiés par les ménages ou encore les différentes stratégies mises en place pour faire face à des chocs ont également été collectées.

Nous avons ensuite procédé à une analyse des revenus, qui se base sur une reconstitution des revenus associés à toutes les activités du ménage, en intégrant l'autoconsommation au prix du marché. Le calcul des revenus comprend à la fois les revenus agricoles (valeur de la production vendue + valeur de l'autoconsommation - charges de production), les revenus des activités d'élevage (valeur de la production vendue + valeur de l'autoconsommation - charges), les revenus des activités extra-agricoles (commerce, salariat, artisanat, pêche...), les transferts des migrants, les aides publiques, les revenus fonciers (issus de la mise en location des parcelles) et le salariat agricole.

\subsubsection{Vers une typologie des ménages}

Pour résumer la grande hétérogénéité et capter les différents facteurs de différenciation de la population observée, nous avons choisi de construire notre typologie en combinant une approche structurelle avec une approche fonctionnelle (Bélières et al., 2017; Tittonell et al., 2020) et en utilisant séquentiellement deux techniques statistiques multivariées (Piron et al., 2004) : l'analyse en correspondance principale (ACP) et une classification ascendante hiérarchique $(\mathrm{CAH})$. Les variables sont centrées et réduites pour homogénéiser les unités et accorder à chaque variable la même importance. La rotation orthogonale varimax a été utilisée. Pour le regroupement, nous avons utilisé la procédure hiérarchique de Ward, permettant d'agréger les individus qui font le moins varier l'inertie interclasse pour obtenir un nombre optimal de grappes (Álvarez et al., 2014). Au départ, 
nous avons sélectionné 46 variables et avons procédé à l'analyse de la matrice de corrélation de Pearson pour déterminer les variables ayant une forte corrélation. Pour chaque paire de variables ayant une forte corrélation, une d'entre elles est supprimée pour éviter la redondance et augmenter le poids apporté par les autres variables discriminantes (Álvarez et al., 2014). Le choix de la variable à supprimer s'est fait en veillant à ce que les variables qui représentent les différents capitaux des moyens d'existence et des stratégies de diversification des sources de revenus soient représentées. La construction de la typologie a porté sur 9 variables et sur 188 ménages, qui correspondent à $91 \%$ de l'échantillon total. Cette analyse typologique a été réalisée en utilisant le logiciel Xlstat.

\section{Résultats}

\subsection{Histoire du socio-écosystème de Guédé}

L'analyse de la socio-histoire nous a permis d'identifier six périodes marquantes.

Période 1. Avant 1930, date d'introduction de la culture irriguée. Le territoire abritant l'actuelle cuvette de Guédé Chantier était une plaine inondée peuplée d'espèces arbustives et herbacées variées. Les habitants vivaient de cultures traditionnelles réparties sur trois espaces différents. Dans le «Walo», inondé naturellement par la crue annuelle du fleuve, situé dans les cuvettes argileuses, étaient cultivés du sorgho et des cultures maraîchères. Les hautes terres sableuses situées hors du lit du fleuve et arrosées par les eaux pluviales, appelées «Diéri», accueillaient les cultures traditionnelles comme le mil, le niébé ou le béréf (Citrullus vulgaris) et abritaient de vastes zones de pâturage. Les «Falo», berges situées sur le lit majeur du fleuve et inondées naturellement par les crues, permettaient la production de légumes et légumineuses.

Période 2 (1930-1960). Sur un potentiel exploitable de 600 ha, le gouvernement colonial aménage 180 ha pour introduire la riziculture irriguée. Les forêts autour de Guédé (sur les terroirs villageois de Lérabé et Guédé village) sont déboisées, les premiers travaux ayant lieu vers 1933. Le colonisateur, aidé des chefs de cantons voisins, recrute de force 50 personnes appelées «colons» et leurs familles, qui sont souvent les plus démunis ou les plus «turbulents». Le village de Guédé Chantier est créé avec l'arrivée de ces colons installés sur 68 ha de riz, chaque colon recevant 0,5 ha de rizière par membre de sa famille adulte pour une année, avec un minimum de 1 ha. En 1943, la conduite des premiers essais de culture du riz et les bonnes récoltes obtenues attirent massivement d'autres personnes volontaires et leurs familles. Mais progressivement, devant la précarité des aménagements et l'envahissement des parcelles par les mauvaises herbes, les rendements finissent par baisser fortement.

Période 3 (1960-1970). Juste après l'Indépendance, l'État prône une logique économique d'autosuffisance alimentaire encadrée. L'Organisation autonome de la vallée (OAV) mise en place en 1961 améliore le modèle d'aménagement basé sur une maîtrise partielle de l'eau par la submersion contrôlée initiée par le colonisateur. Ainsi, les aménagements de types secondaires avec des diguettes internes, des canaux primaires et secondaires remplacent les aménagements primaires.

Période 4 (1970-1980). Les années 1970 sont celles des épisodes de sécheresse qui frappent le Sahel. L'agriculture
Tableau 1. Répartition des périodes d'installation des ménages interrogés.

Table 1. Distribution of settlement periods of surveyed households.

\begin{tabular}{ll}
\hline Période d'installation & Effectifs \\
\hline Avant 1930 (période 1) & 29 \\
Entre 1930 et 1960 (période 2) & 67 \\
Entre 1960 et 1970 (période 3) & 47 \\
Entre 1970 et 1980 (période 4) & 36 \\
Entre 1980 et 1990 (période 5) & 10 \\
Depuis 1990 (période 6) & 17 \\
\hline
\end{tabular}

irriguée rendue possible à Guédé attire une deuxième vague de migrants. De 1969 à 1973, 118 ha sont aménagés dans la cuvette et des techniques de pépinière et de repiquage sont introduites. De 1973 à 1978, 23 ha supplémentaires sont aménagés, d'où un total de 141 ha. En 1978, dans une perspective de diversification agronomique, la culture de la tomate est introduite pour la première fois à Guédé.

Période 5 (1980-1990). Sous la pression des Programmes d'ajustement structurel (PAS) de la Banque mondiale et du Fonds monétaire international, l'État se désengage et libéralise le marché. Une nouvelle politique agricole (NPA) soutient l'intensification pour limiter l'impact des aléas climatiques. En 1984, les Groupements d'intérêt économique (GIE) sont créés et le PIV de Lérabé est aménagé. En 1987, la Caisse nationale de crédit agricole du Sénégal (CNCAS) est créée pour permettre aux paysans d'accéder aux crédits indispensables face aux coûts engendrés par les impératifs de la mécanisation et l'intensification de l'agriculture. En 1989, le PIV de Guédé village est créé.

Période 6 (depuis 1990). La réhabilitation de la cuvette de Guédé s'accompagne d'une nouvelle organisation structurée autour des GIE. Au début des années 1990, l'État réhabilite le périmètre (fortement dégradé) de la cuvette, entraînant l'arrêt total de toute production entre 1992 et 1994. Après la réhabilitation, une nouvelle répartition des terres prend en compte les nouveaux arrivants. Avant la réhabilitation, des négociations entre les détenteurs de terre les ont amenés à décider collectivement de céder $10 \%$ de leurs terres. Ce sont ces terres ainsi libérées et celles nouvellement aménagées qui ont été mises à la disposition de ceux qui n'en avaient pas. La gestion des infrastructures hydrauliques, leur entretien, leur surveillance et le respect des délais de paiement de la redevance sont maintenant assurés par les producteurs organisés en GIE.

Le tableau 1 indique la répartition des périodes d'installation des ménages actuellement présents. Elle illustre le pic de migration qui a suivi l'introduction de la culture irriguée. Cette vague de migration s'est poursuivie jusqu'aux périodes marquées par des épisodes de sécheresses successives. Avec la pression démographique et la faiblesse des rendements, la migration s'est progressivement réduite au fil des années.

L'historique de l'aménagement de la cuvette montre que la forte attraction pour l'agriculture possible toute l'année a eu pour conséquence une saturation foncière, comme le témoigne cet interviewé : «La population augmente alors que les terres n'augmentent pas» (Entretien 6_2019). En effet, dans la cuvette, la configuration pensée par le colonisateur, puis prolongée par l'État sénégalais, a enfermé les agriculteurs dans 
Tableau 2. Caractérisation des types de ménages par axe.

Table 2. Characterization of household types by axis.

\begin{tabular}{|c|c|c|c|c|c|c|c|c|c|}
\hline $\operatorname{Axe}(\mathrm{ACP})$ & Variable & $\begin{array}{l}\text { Contribution de } \\
\text { la variable (\%) }\end{array}$ & $\begin{array}{l}1 \\
(n=33)\end{array}$ & $\begin{array}{l}2 \\
(n=65)\end{array}$ & $\begin{array}{l}3 \\
(n=39)\end{array}$ & $\begin{array}{l}4 \\
(n=23)\end{array}$ & $\begin{array}{l}5 \\
(n=11)\end{array}$ & $\begin{array}{l}6 \\
(n=9)\end{array}$ & $\begin{array}{l}7 \\
(n=8)\end{array}$ \\
\hline \multirow{3}{*}{ Axe 1} & SAU irriguée & 39 & & $\checkmark$ & & $\checkmark$ & & & \\
\hline & Superficie cultivée en irrigué & 30 & & & & $\checkmark$ & & & \\
\hline & Organisations paysannes & 25 & & & & $\checkmark$ & & & \\
\hline Axe 2 & Nombre de personnes & 53 & & & $\checkmark$ & $\checkmark$ & & & \\
\hline \multirow[b]{2}{*}{ Axe 3} & Cheptel total & 63 & & $\checkmark$ & & & & $\checkmark$ & \\
\hline & Équipements & 20 & & & & & & $\checkmark$ & \\
\hline Axe 4 & Ratio bœufs/cheptel total & 45 & & & & & & $\checkmark$ & \\
\hline Axe 6 & Superficie cultivée en non irrigué & 72 & & & & & $\checkmark$ & & \\
\hline Axe 7 & Revenu extra-agricole & 90 & & & & & & & $\checkmark$ \\
\hline \multirow{2}{*}{ Axe 8} & Période installation & 50 & $\checkmark$ & & & $\checkmark$ & $\checkmark$ & & \\
\hline & Équipements & 25 & $\checkmark$ & & & & $\checkmark$ & $\checkmark$ & \\
\hline
\end{tabular}

$\checkmark$ signifie que la coordonnée sur cet axe est forte.

une situation qui limite toute possibilité d'extension. Ainsi, dans le système irrigué, la superficie moyenne possédée par ménage est de 0,6 ha, soit 0,12 ha par actif, alors que dans le delta du fleuve Sénégal, les superficies moyennes par actif sont de l'ordre de 1,2 ha. $15 \%$ des ménages sont sans terres tandis que $20 \%$ des ménages concentrent $90 \%$ de la superficie totale irriguée.

\subsection{Typologie socio-historico-économique}

Les résultats de l'ACP montrent que 8 composantes principales expliquent $80 \%$ de la variation. Elles ont été utilisées pour la classification ascendante hiérarchique.

La CAH a permis d'identifier 7 groupes de ménages qui se distinguent en fonction du nombre d'actifs agricoles, de la superficie agricole utile (SAU) totale irriguée, de la superficie totale cultivée en irrigué et en non irrigué, du nombre d'équipements, du cheptel total, et du revenu extra agricole par actif (Tab. 2).

La SAU irriguée correspond à toutes les parcelles disponibles et utilisables par le ménage dans les différents périmètres irrigués. La superficie cultivée en irrigué correspond à la superficie de toutes les parcelles situées dans les périmètres irrigués que le ménage a cultivées sur toute l'année. La variable nombre d'équipements comptabilise uniquement les charrettes, les ânes et chevaux que possède le ménage. Les principaux équipements agricoles qui entrent dans le processus de production, comme les motopompes ou les tracteurs, sont gérés par les GIE.
Les sept types se caractérisent comme suit:

- Type 1-«Exploitants moyens récemment installés ». Dans ce type, l'installation la plus récente commence sur la période 3 , avec $50 \%$ des ménages installés entre la période 5 et la période 6 . Ces ménages exploitent plus de terres irriguées $(0,8 \mathrm{ha})$ qu'ils n'en possèdent $(0,6 \mathrm{ha})$ et les combinent avec un peu d'agriculture dans les espaces traditionnels $(0,08 \mathrm{ha})$. Ils sont faiblement dotés en cheptel (6 têtes).

- Type $2-\ll$ Petits exploitants faiblement dotés en facteurs de production ». La superficie agricole possédée en irrigué par ménage est la plus faible ( 0,3 ha) et ces ménages sont également les moins dotés en équipements $(0,5)$ et en cheptel (5 têtes).

- Type 3-«Exploitants à faible revenu extra-agricole». Avec le revenu extra-agricole par actif le plus faible (31 068 FCFA, 1000 FCFA = 1,5 $€$ ), les ménages de ce type comptent le plus de personnes (12 personnes). Ils possèdent un cheptel de 9 têtes, essentiellement des petits ruminants $(89 \%)$

- Type 4-«Grands exploitants anciennement installés ». $95 \%$ se sont installés avant 1960 . Ce type se caractérise par les dotations en terres irriguées les plus importantes (1,9 ha) et par la taille de la superficie agricole cultivée en irrigué la plus importante (1,5 ha). Ce sont eux qui appartiennent le plus à des organisations paysannes $(1,7)$.

- Type 5-«Exploitants traditionnels ». Ils se distinguent par la superficie cultivée dans les parcelles traditionnelles (Walo et berges) la plus importante (2,3 ha). Ils sont 


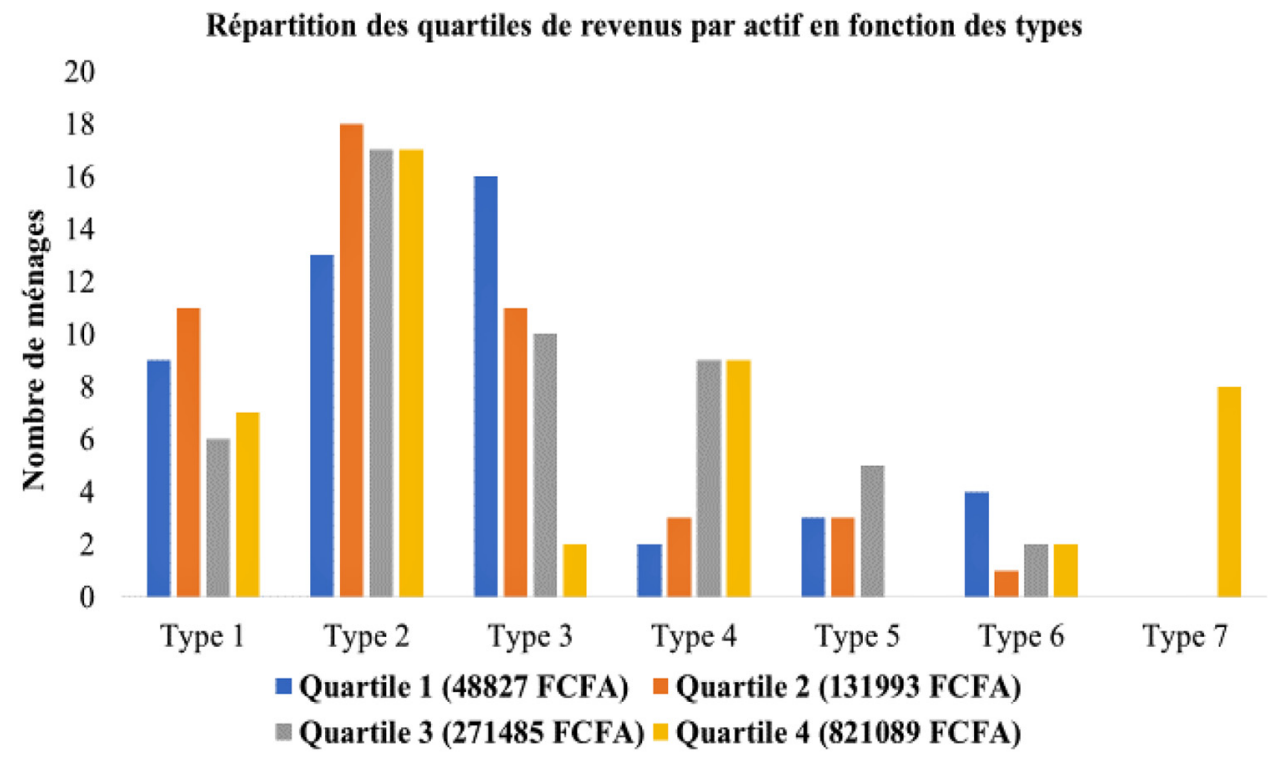

Fig. 2. Répartition des différents types dans les quartiles de revenus.

Fig. 2. Distribution of the different types in income quartiles.

également bien dotés en équipements $(2,7)$ et très peu engagés dans les organisations sociales $(0,1)$.

- Type 6-«Éleveurs ». Ils sont peu dotés en terres (0,3 ha). $\mathrm{C}$ 'est le type qui cultive le moins de superficies en irrigué $(0,4$ ha) et qui appartient le moins à des organisations paysannes $(0,5)$. Il se distingue par ses dotations en cheptel les plus importantes (48 têtes) et est également le type qui compte le plus d'équipements $(3,3)$.

- Type 7-«Ménages extra-agricoles ». Ils se distinguent par leurs revenus extra-agricoles par actif les plus importants (534 132 FCFA). Ce type est le deuxième le plus doté en terres irriguées ( $0,8 \mathrm{ha}$ ) et en bétail (27 têtes), mais exploite peu de terres en irrigué $(0,5 \mathrm{ha})$ et n'exploite aucune surface dans les espaces traditionnels.

\subsection{Analyse du profil des revenus des ménages dans les 7 types}

L'analyse du profil des revenus des ménages à travers la méthode des quartiles (Faye et al., 2021) nous a permis d'analyser la distribution des revenus par tranche de $25 \%$ des ménages étudiés et de visualiser la relation entre la distribution des revenus nets par actif et les types identifiés (Fig. 2). Elle montre que les deux premiers quartiles sont situés en dessous du seuil de pauvreté global par adulte au Sénégal, évalué selon l'Agence nationale de la statistique et de la démographie (ANSD) à 218000 FCFA par an (ANSD, 2013). Ramené à l'échelle du consommateur, seul le type 7 se retrouve au-dessus du seuil de pauvreté par personne. Cette première analyse révèle les inégalités de revenu et la forte pauvreté qui touche la majorité des ménages du système irrigué de Guédé.

Le premier élément de différenciation des revenus est l'opposition assez marquée entre le type 3, caractérisé par de faibles revenus extra-agricoles, qui se trouve majoritairement (47\%) dans le quartile le plus faible, et le type 7 , qui cultive peu de terres et est caractérisé par de forts revenus extra agricoles, qui se trouve entièrement $(100 \%$ des ménages concernés) dans le quartile le plus élevé. Les ménages du type 7 , très orientés vers les revenus extra-agricoles, et les ménages du type 4, qui sont les grands propriétaires de terres et qui cultivent le plus, se retrouvent majoritairement au-dessus du seuil de pauvreté par actif.

\subsection{Les facteurs de stress}

La figure 3 représente les réponses des ménages interrogés à la question des facteurs de stress. Le stress dominant pour les types $1,2,3,5$ et 7 est la faiblesse des rendements. Le type 2, le plus faiblement doté en ressources, est celui qui déclare le plus de facteurs de stress (notamment financiers). Le type 4, plus spécialisé dans l'agriculture, rencontre plus de difficultés liées à la commercialisation ou à l'endettement. Le type 6 , spécialisé dans l'élevage, a déclaré un seul facteur de stress, lié à la sécheresse.

L'analyse à dire d'acteurs montre que les enquêtés ont très souvent évoqué le lien direct entre la faiblesse des rendements observés dans les périmètres et l'appauvrissement des sols. «... Avant, tu cultivais 5 ares, tu avais 50 sacs, maintenant si tu cultives 5 ares, si Dieu ne t'aide pas, tu n'auras pas 4 sacs, parce que la terre est fatiguée» (Entretien 3_2019). Ce lien a été abordé de manière spontanée et systématique par la plupart des agriculteurs interrogés.

\section{Analyse des résultats au prisme de la vulnérabilité des ménages}

\subsection{Stratégies des agriculteurs}

Pour analyser les stratégies des ménages pour faire face aux stress et à la vulnérabilité, nous avons observé un certain nombre de variables supplémentaires (Tab. 3).

L'analyse globale montre une hétérogénéité des situations et des stratégies des ménages : les différents types identifiés ont tous accès à l'irrigation et sont tous engagés dans des stratégies 


\section{Répartition des facteurs de stress par type}

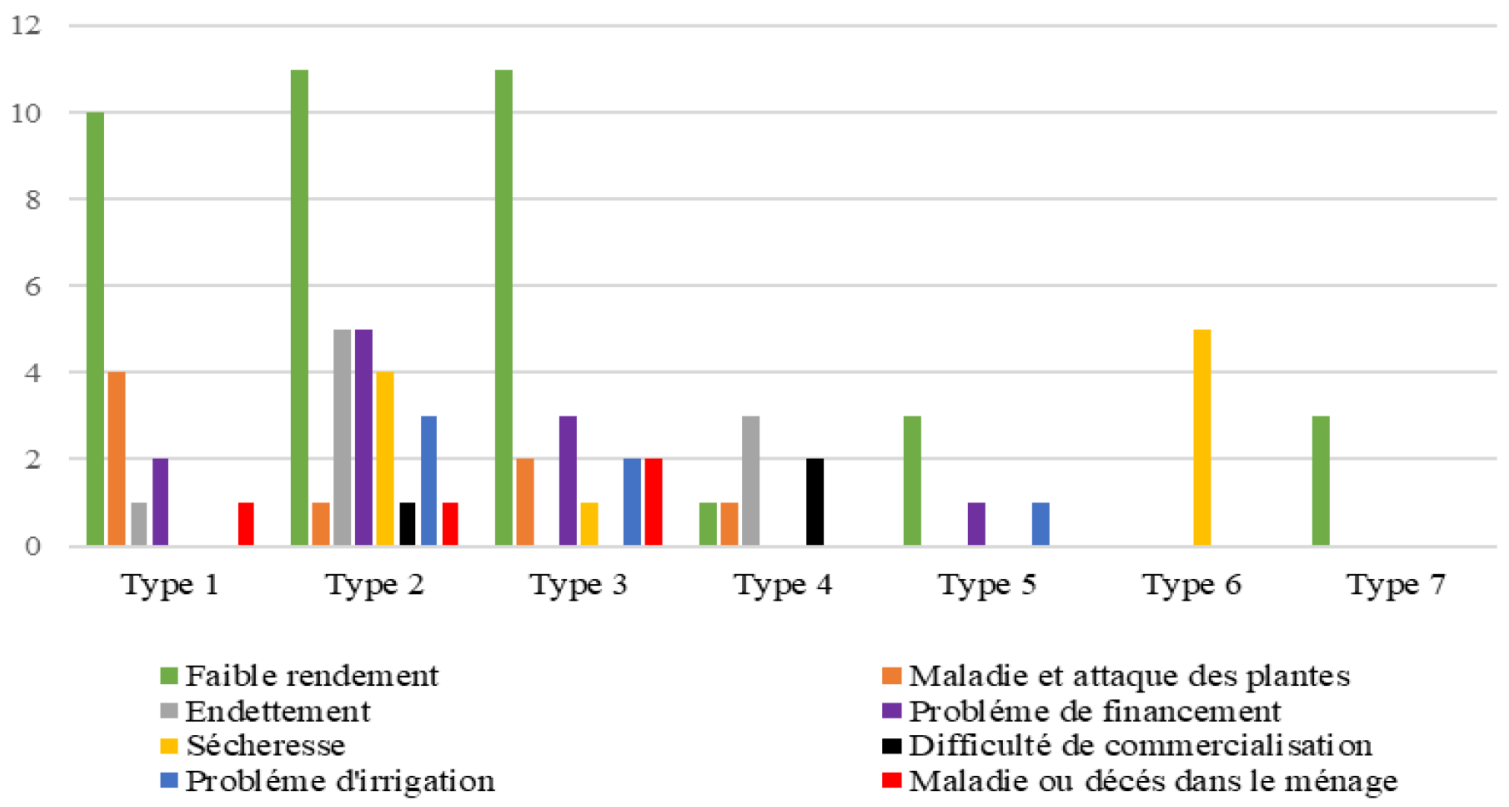

Fig. 3. Facteurs de stress marquants par type.

Fig. 3. Significant stressors by type.

Tableau 3. Caractéristiques des stratégies de diversification des activités et des revenus en fonction des types.

Table 3. Characteristics of activity and income diversification strategies by type.

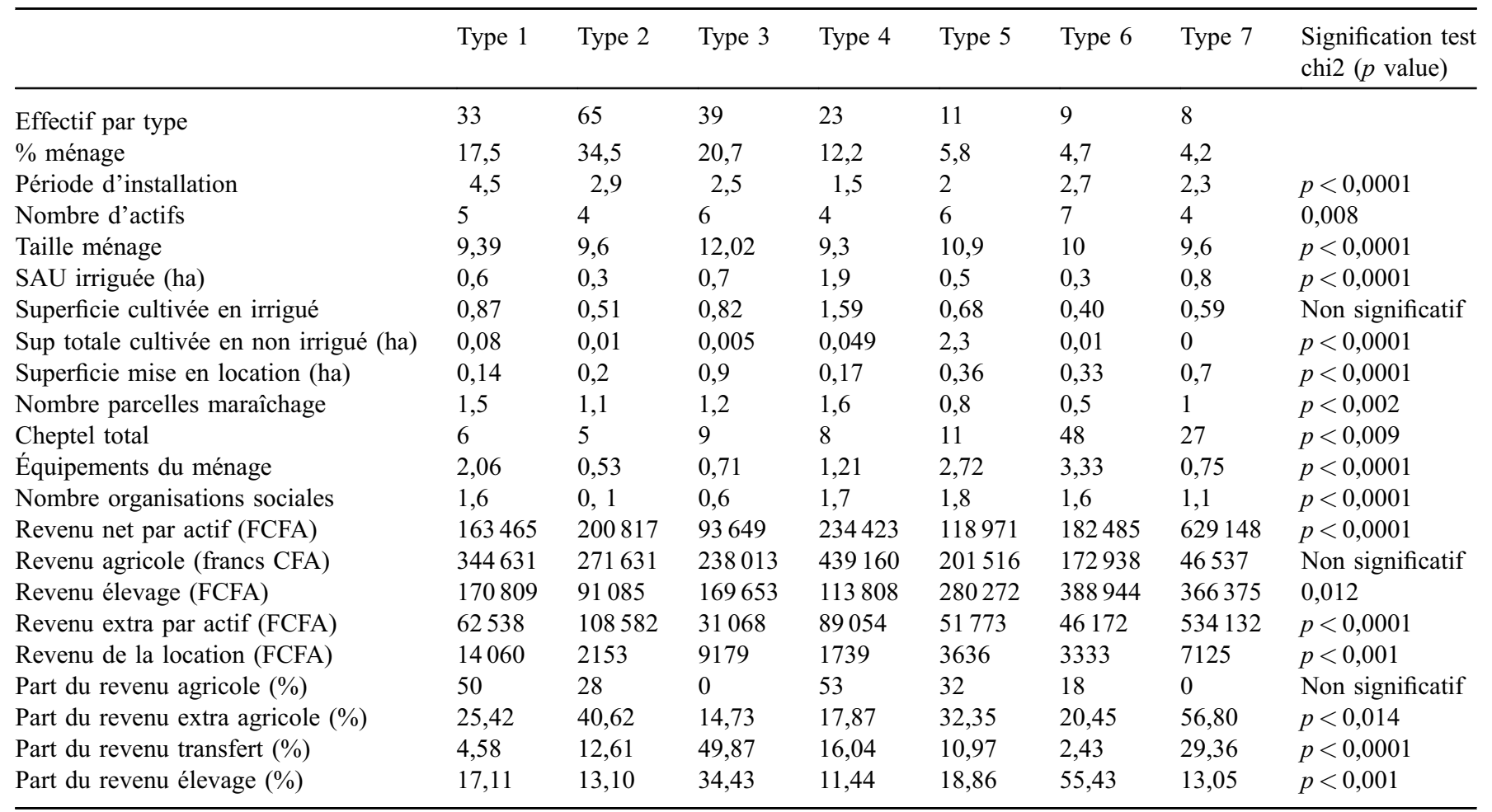


de diversification différentes, qui peuvent s'apprécier à travers la diversité des activités et des sources de revenus selon le type. Le choix du type de stratégie est déterminé par les opportunités ou les contraintes dans l'accès aux ressources.

Le type 1, plus récemment installé et bénéficiant de très peu de revenus de transfert $(4,5 \%)$, est le plus engagé dans les organisations sociales. L'essentiel de ses revenus provient de l'agriculture $(50 \%)$ et il oriente davantage ses stratégies agricoles vers le maraîchage, dont il cultive plusieurs parcelles (1,5 parcelle). Même si ce n'est pas le plus doté en terres, il diversifie ses revenus en mettant certaines de ses parcelles en location, d'où ses revenus fonciers les plus importants (14060 FCFA).

Le type 2, faiblement doté en ressources et moins engagé dans les organisations sociales, mobilise davantage ses actifs dans les activités extra-agricoles, qui représentent $41 \%$ du revenu total du ménage. Très peu doté en terres $(0,3 \mathrm{ha})$, il bénéficie des revenus fonciers les plus faibles (2153 FCFA).

Le type 3 tire l'essentiel de ses revenus des transferts des migrants de la famille, ce qui correspond à $50 \%$ de son revenu total. Il oriente également ses stratégies vers la vente d'animaux, qui représente $34 \%$ de son revenu total. Il ne tire pas de revenus de ses activités agricoles et est celui qui a le moins recours aux activités extra-agricoles, qui correspondent à $15 \%$ de son revenu. Le revenu net par actif pour ce type est le plus faible (93 649 FCFA).

Le type 4, plus anciennement installé, fait partie de la tranche qui a le moins d'actifs (4 actifs). Le plus doté en terres irriguées, il oriente davantage ses stratégies vers l'agriculture, d'où il tire la moitié de ses revenus $(53 \%)$.

Le type 5, moins engagé dans les organisations sociales $(0,1)$, oriente davantage ses stratégies vers l'agriculture et les activités extra-agricoles. Il favorise la combinaison d'agriculture irriguée et d'agriculture traditionnelle (2,3 ha). Le revenu agricole, de même que le revenu extra-agricole, participent chacun à $32 \%$ du revenu global du ménage. Il se dote d'équipements (charrettes, chevaux...) pour assurer ses déplacements vers les espaces traditionnels, qui sont plus éloignés que les périmètres irrigués aménagés.

Le type 6 est celui qui appartient le moins à des organisations paysannes et bénéficie le moins de transferts de migrants $(2,43 \%$ du revenu total). Le plus doté en cheptel (48 têtes), il oriente davantage ses stratégies vers la vente d'animaux, qui représente $55 \%$ de son revenu global. Il se dote ainsi d'équipements $(3,3)$ lui permettant d'assurer ses déplacements vers les marchés pour vendre des animaux. Même s'il est moins doté en terres que le type 3 et le type 7 , il tire plus de revenus de ses activités agricoles, qui correspondent à $18 \%$ de son revenu global. C'est le type qui compte le plus d'actifs ( 7 actifs) et il les mobilise dans les activités extraagricoles, d'où la part non négligeable du revenu extra-agricole $(20 \%)$.

Le type 7 n'exploite aucune parcelle en culture traditionnelle et ne tire pas non plus de revenus de ses activités agricoles. L'essentiel de ses revenus provient des activités extra-agricoles que sont le commerce, le salariat agricole, la pêche, la transformation et des professions fixes (enseignement, administration). Il bénéficie également de transferts des membres de la famille (migrants) qui représentent une part assez importante de son revenu global (29\%).

\subsection{Effets combinés de l'histoire et de la géographie sur la vulnérabilité actuelle des ménages}

L'analyse croisée des périodes d'installation des ménages par village et la répartition des types par village confirme la relation entre les types, les villages et leur période d'installation (Fig. 4).

Les ménages les plus anciennement installés sont à Guédé Village. Les ménages de Médina Fresbé sont arrivés aux périodes 2 et 3 . Les ménages se sont installés en continu à Agnam Tonguel, sauf pour la période la plus récente. L'installation à Guédé-Chantier est continue, comme à Lérabé depuis la période P3.

Les ménages des types 1,2 et 3 se retrouvent un peu partout, comme les ménages de type 7 (sauf à Lérabé). Ils sont très majoritaires à Guédé Chantier. Les ménages des types 4 et 5 sont majoritaires à Guédé Village et les ménages du type 6 sont à Médina Fresbé.

\section{Discussion et conclusion: construction et différenciation de la vulnérabilité}

Bien que les systèmes irrigués augmentent la capacité de production et limitent la sensibilité vis-à-vis de la sécheresse, la population croissante (issue de l'immigration ou du croît démographique), attirée par l'irrigation, et la concentration spatiale remettent en question la capacité des ressources à couvrir les besoins des ménages (Nelson et al., 2010). Avec la fragmentation foncière au fil des générations, qui entraîne une diminution de la taille des exploitations, et l'absence d'opportunités pour accéder à d'autres espaces irrigués, nos données montrent que les superficies détenues par ménage sont trop faibles (Tab. 3) pour couvrir les besoins alimentaires et monétaires des ménages. Cela impose un système de culture intensif qui nécessite l'utilisation de produits chimiques. L'analyse à dire d'acteurs et de la littérature (Poussin et al., 2002) montre que la surutilisation des terres perturbe leur régénération naturelle et entraîne à long terme la pauvreté des sols; la forte utilisation des fertilisants peut contribuer à la pollution des eaux de surface et souterraines. Cette pression sur les terres entraîne la faiblesse des productions et la dégradation de l'environnement (Headey et Jayne, 2014). De plus, le déboisement pour aménager les périmètres a entraîné la disparition de certaines ressources ligneuses, ce qui constitue une perte d'options de revenus pour les ménages, entraînant une pression supplémentaire sur les ressources disponibles.

La typologie révèle une hétérogénéité des ménages, qui est fonction des caractéristiques structurelles et fonctionnelles des ménages, mais aussi de leur période et lieu d'installation.

L'analyse des revenus montre une forte pauvreté qui touche la quasi-totalité des ménages $(95 \%)$. Seul le type 7 se trouve au-dessus du seuil de pauvreté par consommateur. Les ménages qui s'en sortent le mieux économiquement sont ceux qui privilégient les activités extra-agricoles et ceux qui ont des superficies agricoles irriguées importantes. Or, seuls $3 \%$ des ménages possèdent plus de 0,5 ha par actif.

Chaque type présente différentes expositions et sensibilités et développe différentes adaptations en fonction des ressources dont il dispose (Tab. 4). 


\section{Répartition des périodes d'installations et des types par village}
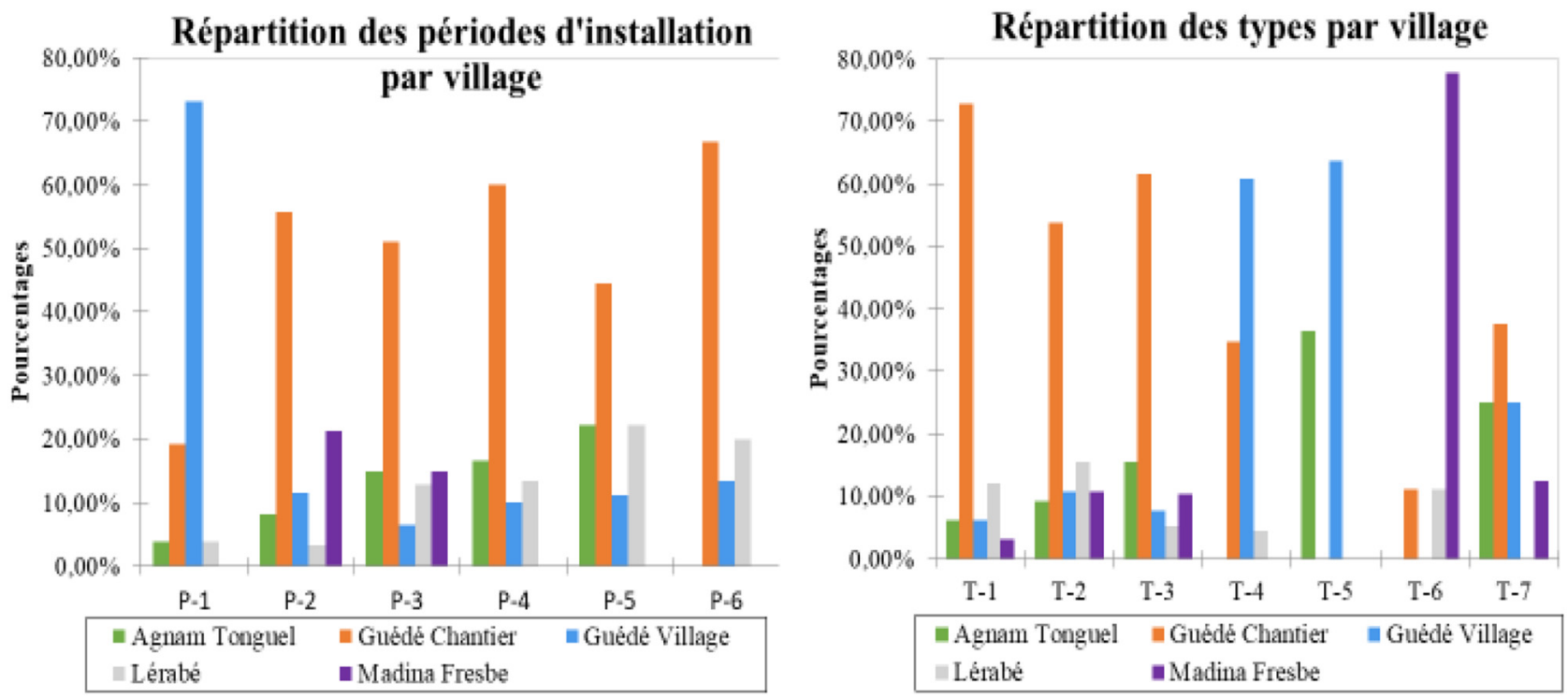

La figure de Gauche montre la répartition des périodes d'installations par village . La figure de droite montre la répartition des types par village

$\mathrm{p}=$ Période

$\mathrm{T}=$ Type

Notes : Les périodes vont de la période 1 (P1), période d'installation la plus ancienne à la période 6 (P6), période d'installation la plus récente. Plus $\mathrm{P}$ est petit, plus la période d'installation est ancienne, et plus $\mathrm{P}$ est grand plus la période d'installation est récente.

Fig. 4. Répartition des périodes d'installation et des types par village.

Fig. 4. Distribution of installation periods and types by village.

Tableau 4. Stress et stratégies en fonction des types.

Table 4. Stress and strategies according to types.

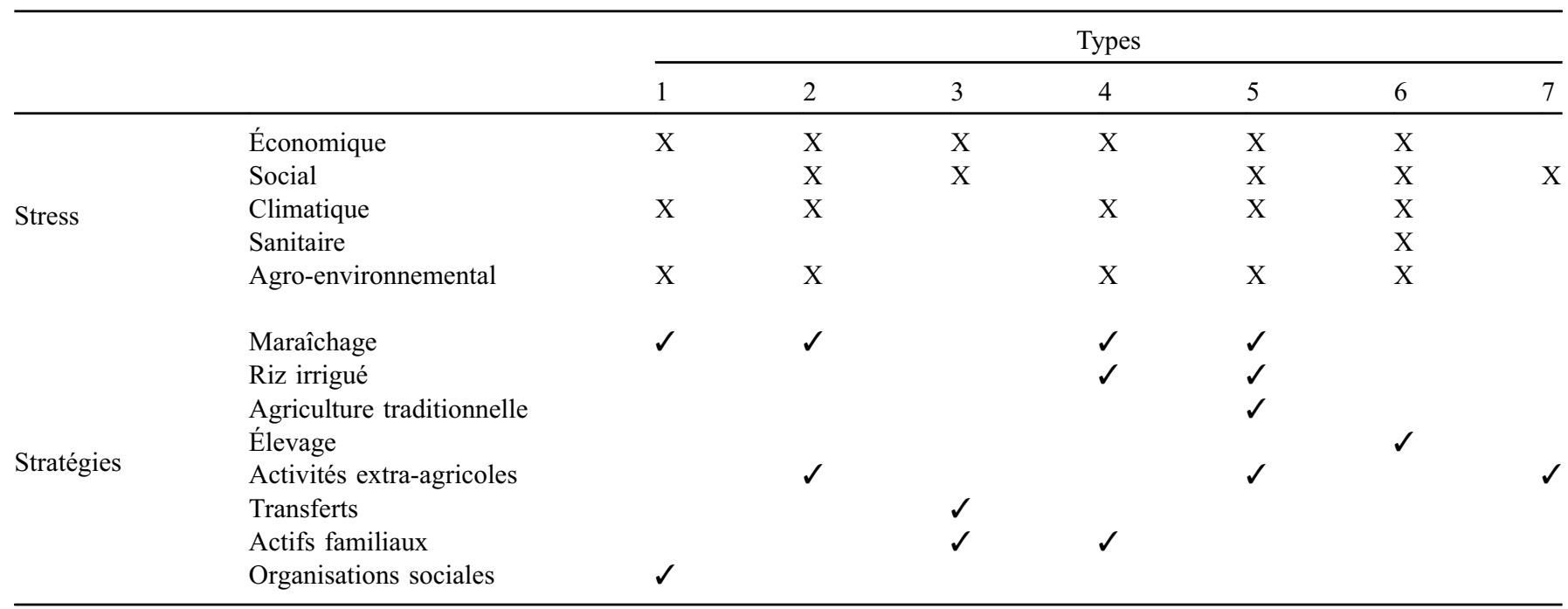

Dans notre analyse, nous avons caractérisé l'exposition et la sensibilité en considérant les différents stress soulevés par les personnes interrogées. Les ménages du type 6, majoritaires à Madina Fresbé, bien dotés en ressources animales et dont les stratégies sont orientées vers l'élevage, se déclarent exposés et sensibles aux stress liés à la sécheresse, qui entraînent une pénurie d'aliments pour le bétail (voir Fig. 3), ou aux maladies qui s'attaquent au bétail. L'épidémie de fièvre de la vallée du Rift dans les années 1990 et des pluies hors saison en 2002 ont conduit à la mort de la majeure partie du bétail. Ces événements extrêmes ont placé les ménages dans une situation de pauvreté dont ils peinent toujours à se relever. 
Les résultats des enquêtes et des entretiens révèlent une forte sensibilité à la chaleur des ménages qui dépendent beaucoup de la production végétale, notamment pour le maraîchage où la chaleur provoque des brûlures des plants de tomate et le blocage de la croissance de la plante. Ils montrent aussi que les producteurs souffrent d'un problème de commercialisation des produits de la récolte, qui finissent parfois par pourrir. Cela résulte d'un mauvais fonctionnement du marché, qui peine à se réguler, si bien que les productions agricoles pâtissent d'une instabilité des prix considérable. Cela est notamment observé pour l'oignon. La concurrence de l'oignon importé, l'absence d'infrastructures de conservation et de transformation font que les producteurs sont sous la dépendance des «banabanas », qui sont des acheteurs en demigros ou au détail auprès des producteurs pour revendre aux commerçants ou aux consommateurs dans les marchés, et qui fixent leurs prix selon la production. Ainsi, il n'est pas rare de vendre son sac d'oignon à un prix trois ou quatre fois inférieur au prix du marché de détail.

Les stratégies d'adaptation sont façonnées par l'histoire (période d'installation) et la géographie (lieu d'installation). Les ménages anciennement installés (Type 4) de Guédé Village et Guédé Chantier sont plus orientés vers l'agriculture irriguée (riz et maraîchage) et l'agriculture traditionnelle (Type 5). Les ménages récemment installés (Type 1) orientent davantage leurs stratégies agricoles vers le maraîchage, alors que les ménages du type 6 , installés uniquement sur la période 2 et 3 et majoritaires à Madina Fresbé, sont plus orientés vers l'élevage.

L'analyse nous montre que la diversification des activités et des actifs ne garantit pas une sécurité économique. Les types avec une diversification prononcée n'ont pas forcément les revenus les plus importants (Type 6). A contrario, le type 7 qui a une stratégie d'adaptation orientée sur les activités extraagricoles et le type 4 qui a une stratégie majoritairement orientée sur l'agriculture sont les types où les ménages s'en sortent le mieux économiquement.

Le fonctionnement du système irrigué de Guédé résulte d'une multitude de facteurs dynamiques : la vulnérabilité est la conjonction de facteurs sociaux, historiques, économiques, géographiques et environnementaux interdépendants. La vulnérabilité des ménages s'est construite au cours des transformations socio-écologiques qu'a connues le système irrigué depuis la période coloniale jusqu'à nos jours. La vulnérabilité différentielle des ménages tient à la période et au lieu d'installation, qui orientent l'accès et la structure des ressources. Cela définit les capacités des ménages à convertir ces ressources en activités et en revenus stratégiques pour survivre à la faiblesse des moyens d'existence dans des conditions détériorées, ainsi qu'à la sécurité des moyens d'existence dans des conditions économiques améliorées (Ellis, 2000; Adger, 2006).

L'approche socio-historico-économique postule que la vulnérabilité est une construction qui se déploie sur le temps long, au fur et à mesure que les sociétés aménagent et modifient un territoire (Antoine, 1992). Les résultats de ce travail montrent que les différents types de ménages, fortement façonnés par la période et le lieu d'installation, leurs ressources et leurs revenus, présentent des vulnérabilités différentielles à plusieurs facteurs de stress. Ce travail illustre le lien fort qui existe entre la vulnérabilité et l'histoire, la géographie, l'accès aux ressources, les activités des moyens d'existence et la dégradation de l'environnement. Comme l'ont montré les travaux de Eakin et al. (2009) et O'Brien et al. (2009), pour comprendre la vulnérabilité et les implications de l'adaptation, il est nécessaire d'éclairer les interactions complexes de cause et conséquence des actions et décisions à différentes échelles spatiales et temporelles. L'étude démontre que le processus historique de mise en place des infrastructures hydro-agricoles à Guédé a profondément transformé les conditions d'accès aux ressources (terre, eau) par les groupes sociaux. Les dotations différenciées ont conditionné et conditionnent encore les opportunités et les contraintes distinctes des moyens d'existence entre ménages agricoles et expliquent leurs vulnérabilités différentes face à des chocs internes et externes. En fonction des chocs auxquels ils sont confrontés, ces ménages ont des capacités inégales à développer des stratégies d'adaptation efficaces.

Les résultats de nos travaux pourraient être comparés à ceux obtenus par Barbier et al. (2011), Jamin et al. (2011), Bélières et al. (2013) et Poussin et al. (2015).

Face au débat controversé sur la contribution des systèmes irrigués à l'amélioration des conditions d'existence des populations (Adams, 2006; Le Roy, 2006; Jamin et al., 2011), la mobilisation d'une approche socio-historico-économique nous a permis de considérer plusieurs dimensions qui donnent des clés de lecture sur la construction de la vulnérabilité et de comprendre pourquoi et comment des vulnérabilités spécifiques se produisent à des moments spécifiques, à des endroits spécifiques et pour des personnes spécifiques.

Au-delà du discours voulant afficher l'irrigation comme solution unique pour la sécurisation de la production et l'augmentation des revenus, nous pouvons affirmer que dans le système irrigué de Guédé, l'irrigation à elle seule n'assure pas la sécurité des moyens d'existence des ménages. Faciliter la combinaison de l'irrigation à d'autres activités (agriculture traditionnelle ou activités extra-agricoles) ne serait-il pas un moyen de rendre les ménages moins vulnérables?

Cette analyse attire l'attention sur l'intérêt de prendre en compte l'hétérogénéité des schémas de vulnérabilité qui peut être essentielle dans la planification de l'adaptation, pour éviter une maladaptation et ainsi renforcer la résilience des populations qui cultivent dans les systèmes irrigués.

Remerciements. Les auteurs adressent leurs remerciements à Montpellier université d'excellence (MUSE), qui a financé ce travail. Les auteurs voudraient aussi remercier Martin Diedhiou pour son appui sur le terrain et toute l'équipe des enquêteurs. Nos vifs remerciements sont adressés également à tous les agriculteurs et à tous les acteurs de terrain qui ont accepté de répondre à nos questions.

\section{Références}

Adams A. 2006. WCD Thematic Review I.1: Contributing PaperSocial impacts of an African dam: Equity and distributional issues in the Senegal River Valley. Working Paper, eSocialSciences. https://econpapers.repec.org/paper/esswpaper/id 3a514.htm.

Adger WN. 2006. Vulnerability. Global Environmental Change 16 (3): 268-281. https://doi.org/10.1016/j.gloenvcha.2006.02.006. 
Álvarez S, Paas W, Descheemaeker K, Tittonell PA, Groot JCJ. 2014. Typology construction: A way of dealing with farm diversity: General guidelines for humidtropics. Wageningen, The Netherlands: Wageningen University. https://cgspace.cgiar.org/handle/ 10568/65374

ANSD. 2013. Rapport définitif de la deuxième Enquête de Suivi de la Pauvreté au Sénégal (ESPS II). [2021/11/19]. http://anads.ansd.sn/ index.php/citations/3.

Antoine JM. 1992. La catastrophe oubliée: les avatars de l'inondation, du risque et de l'aménagement dans la vallée de l'Ariège (Pyrénées françaises, fin $\mathrm{XVII}^{\mathrm{e}}-\mathrm{XX}^{\mathrm{e}}$ siècles). Thèse de doctorat, Université Toulouse 2. http://www.theses.fr/ 1992 TOU20005.

Bankoff G. 2004. Time is of the Essence: Disasters, Vulnerability and History. International Journal of Mass Emergencies and Disasters 22(3): 23-42.

Barbier B, Ouédraogo H, Dembélé Y, Yacouba H, Barry B, Jamin JY. 2011. L'agriculture irriguée dans le Sahel ouest-africain. Cahiers Agricultures 20(1-2): 24-33. https://doi.org/10.1684/ agr.2011.0475.

Bélières JF, Rasolofo P, Rivolala B, Ratovoarinony R, Ratsaramiarina O, Rabevohitra BN, et al. 2017. Élaboration de typologies d'exploitations agricoles au niveau infranational à Madagascar: Lac Alaotra et région du Ménabe. Antananarivo (Madagascar): CIRAD-FAO, $57 \mathrm{p}$.

Bélières JF, Jamin JY, Seck SM, Tonneau JP, Adamczewski A, Le Gal PY.2013. Land dynamics, investments and production models in the West African irrigated sector: Financial rationale against social coherence? Cahiers Agricultures 22(1): 61-66. https://doi.org/ 10.1684/agr.2012.0574.

Bruckmann L. 2018. Crue et développement rural dans la vallée du Sénégal : entre marginalisation et résilience. Belgeo (2). https://doi. org/10.4000/belgeo.23158.

Chambers R, Conway G. 1991. Sustainable rural livehoods: Pratical concepts for the 21st century. IDS Discussion Paper 296, $33 \mathrm{p}$.

Combessie JC. 2007. IV. Sondages, échantillons. Repères 5: 45-54.

Eakin H, Winkels A, Sendzimir J. 2009. Nested vulnerability: exploring cross-scale linkages and vulnerability teleconnections in Mexican and Vietnamese coffee systems. Environmental Science \& Policy 12(4): 398-412. https://doi.org/10.1016/j. envsci.2008.09.003.

Ellis F. 2000. Rural livelihoods and diversity in developing countries. Oxford (Great Britain): Oxford University Press, $300 \mathrm{p}$.

Faye NF, Gérard F, Sall M, Affholder F, Roudier P. 2021. Pauvreté et inégalités en milieu rural dans le centre du Sénégal : état des lieux, causes et conséquences. In: $14^{e s}$ journées de la recherche en sciences sociales, INRAE, SFER, CIRAD, 24 p. https://agritrop. cirad.fr/598426/.

Headey DD, Jayne TS. 2014. Adaptation to land constraints: Is Africa different? Food Policy 48: 18-33. https://doi.org/10.1016/j. foodpol.2014.05.005.

Jamin JY, Bouarfa S, Poussin JC, Garin P. 2011. Les agricultures irriguées face à de nouveaux défis. Cahiers Agricultures 20(1-2): 10-15. https://doi.org/10.1684/agr.2011.0477.
Janssen MA, Schoon ML, Ke W, Börner K. 2006. Scholarly networks on resilience, vulnerability and adaptation within the human dimensions of global environmental change. Global Environmental Change 16(3): 240-252. https://doi.org/10.1016/j.gloenv cha.2006.04.001.

Le Roy X. 2006. Agriculture irriguée et inégalités sociales dans la vallée du fleuve Sénégal PCSI. In: $4^{e}$ Séminaire international et interdisciplinaire, Montpellier, France, $13 \mathrm{p}$. http://hal.cirad.fr/ cirad-00153767/document.

Nelson MC, Kintigh K, Abbott DR, Anderies JM. 2010. The crossscale interplay between social and biophysical context and the vulnerability of irrigation-dependent societies: Archaeology's longterm perspective. Ecology and Society 15(3): 31. https://doi. org/10.5751/ES-03389-150331.

O’Brien K, Quinlan T, Ziervogel G. 2009. Vulnerability interventions in the context of multiple stressors: lessons from the Southern Africa Vulnerability Initiative (SAVI). Environmental Science \& Policy 12(1): 23-32. https://doi.org/10.1016/j.envsci.2008.10.008.

Piron M, Dureau F, Mullon C. 2004. Utilisation de typologies multidates pour 1'analyse des transformations socio-spatiales de Bogota (Colombie). Cybergeo: European Journal of Geography. https:// doi.org/10.4000/cybergeo.3742.

Poussin JC, Boivin P, Hammecker C, Maeght JL. 2002. Riziculture irriguée et évolution des sols dans la vallée du Sénégal. In: Actes de l'atelier $d u$ PCSI, 28-29 mai 2002, Montpellier, France. CEMAGREF, CIRAD, IRD, $8 \mathrm{p}$.

Poussin JC, Renaudin L, Adogoba D, Sanon A, Tazen F, Dogbe W, et al. 2015. Performance of small reservoir irrigated schemes in the Upper Volta basin: Case studies in Burkina Faso and Ghana. Water Resources and Rural Development 6: 50-65. https://doi.org/ 10.1016/j.wrr.2015.05.001

Rurinda J, Mapfumo P, van Wijk MT, Mtambanengwe F, Rufino MC, Chikowo R, et al. 2014. Sources of vulnerability to a variable and changing climate among smallholder households in Zimbabwe: A participatory analysis. Climate Risk Management 3: 65-78. https:// doi.org/10.1016/j.crm.2014.05.004.

Schmitz J. 2008. La vallée du Sénégal entre (co)développement et transnationalisme. Politique africaine 109(1): 56-72. https://doi. org/10.4000/rga.5008.

Spiegelberger T, Bergeret A, Crouzat É, Tschanz L, Piazza-Morel D, Brun JJ, et al. 2018. Construction interdisciplinaire d'une trajectoire socio-écologique de vulnérabilité à l'exemple du territoire des Quatre Montagnes (Isère, France) de 1950 à 2016. Journal of Alpine Research 106(3). https://doi.org/10.4000/ rga.5008.

Tittonell P, Bruzzone O, Solano-Hernández A, López-Ridaura S, Easdale MH. 2020. Functional farm household typologies through archetypal responses to disturbances. Agricultural Systems 178: 102714. https://doi.org/10.1016/j.agsy.2019.102714.

Turner BL, Kasperson RE, Matson PA, McCarthy JJ, Corell RW, Christensen L, et al. 2003. A framework for vulnerability analysis in sustainability science. Proceedings of the National Academy of Sciences 100(14): 8074-8079. https://doi.org/10.1073/ pnas. 1231335100 .

Citation de l'article : Sila A, Gérard F, Daré W, Ba A, Faye E, Adamczewski A, Bousquet F. 2022. Analyse de la construction de la vulnérabilité des ménages du système irrigué de Guédé au nord du Sénégal. Cah. Agric. 31: 6. 